\title{
Memories of the Ebola Virus Disease outbreak affect decisions regarding health care service utilization in Guéckédou district (epicentre) in Guinea: A cross-sectional study of children with febrile illness
}

\section{Bienvenu Salim CAMARA}

Centre National de Formation et de Recherche en Santé Rurale de Maferinyah https://orcid.org/0000-0002-2859-7684

Junko Okumura ( $\sim$ juju3desu@yahoo.co.jp)

https://orcid.org/0000-0002-1744-7824

Alexandre Delamou

Centre National de Formation et de Recherche en Santé Rurale de Maferinyah

\section{Research article}

Keywords: Ebola Virus Disease (EVD), Febrile illnesses, Health seeking behaviour, Rural, Guinea

Posted Date: August 14th, 2019

DOI: https://doi.org/10.21203/rs.2.12713/v1

License: (9) (i) This work is licensed under a Creative Commons Attribution 4.0 International License. Read Full License

Version of Record: A version of this preprint was published on August 27th, 2020. See the published version at https://doi.org/10.1186/s12889-020-09359-0. 


\section{Abstract}

Background The 2013-2015 Ebola Virus Disease (EVD) outbreak in Guinea resulted in community mistrust that influenced health care service utilization. This study aimed to assess whether EVD-related memories affect post-outbreak health-seeking behaviours for children under five years of age with febrile illnesses in Guéckédou district, Guinea. Methods This crosssectional study was conducted by interviewing caregivers of children under five years of age in the sub-district most affected by the EVD outbreak (Guèndembou) and the least affected sub-district (Bolodou) in Guéckédou district. Memories of the outbreak were referred to as EVD-related fears in the post-EVD period, which was based on a series of questions regarding current feelings. Results While the majority of caregivers sought care for their children with febrile illness in both districts, a statistically significantly higher proportion of caregivers in Guèndembou sought care, compared to caregivers in Bolodou. Though not statistically significant, caregivers with a fear score above the median and those who reported the death of family members or friends due to EVD were more likely to seek care. Conclusions This study found that the presence of EVD memories is likely to have a positive influence on health-seeking behaviour in the rural district of Guéckédou. However, the findings call for more efforts to preserve communities' key values and address the psychosocial effects of EVD in rural Guinea.

\section{Background}

The 2013-2015 Ebola virus disease (EVD) outbreak was the widest, longest, and deadliest one ever witnessed worldwide (1). In Guinea, where 3,351 EVD cases were reported - out of which 2,083 (62.2\%) died (2) - the devastating pace of the EVD frightened and confused communities $(3,4)$.

In fact, the inappropriate approach of the early EVD response, which included poor communication about the disease and lack of involvement of community agencies in response activities at the community level, led to a general misconception of the outbreak and mistrust in the health system (5). Indeed, the EVD response required measures that were perceived by the community to be provocative and dismissive of traditional values. For instance, despite being part of local communities' key traditional values, handshaking was discouraged, and community burial or mourning was prohibited $(3,4,6-8)$. Community misconception of the EVD outbreak seemed to cause non-adherence to EVD prevention and control measures. This led to the spread of the outbreak and ravaged families $(3,4,8)$.

Further, people refrained from attending health facilities, fearing that they would be considered EVD cases or be contaminated by the virus $(5,9,10)$. The decline in health service utilization may have been more pronounced for febrile illnesses because fever is the main symptom of EVD. This could be particularly detrimental for malaria patients, especially children who are the most predominantly affected group in Guinea $(11,12)$. Plucinski et al. reported a $15 \%$ decline in public health facility attendance for febrile illnesses and 74,000 fewer malaria cases were treated in Guinea in 2014 compared to 2013 (11).

New approaches, including community involvement in EVD response activities, were later implemented, and they contributed to overcoming the outbreak $(8,10,13)$. For instance, a study in 2015 reported that $62 \%$ of community respondents in Guinea interrupted their practice of traditional initiation ceremonies following implementation of a community-based awarenessraising campaign during the EVD outbreak (14).

Although the EVD outbreak is over in Guinea, communities might still suffer fears related to it. Their tragic human experience of the outbreak $(5,8)$, and the negative effect of the outbreak crisis on their socioeconomic level, ${ }^{14}$ might influence their postoutbreak feelings and behaviours, including their health-seeking behaviours (especially for febrile illnesses). Children's health or chance of survival during early childhood highly depends on appropriate health care service utilisation. Therefore, this age group might be particularly vulnerable to a change in health-seeking behaviour (12).

Little is known about the effects of disease outbreaks on health-seeking behaviour. However, in Mexico, Aguero and Beleche concluded that the $2009 \mathrm{H} 1 \mathrm{~N} 1$ pandemic motivated people into changing their behaviour (washing their hands) and this behaviour change led to a decline in diarrhoea cases for children during and three years after the pandemic (15). In Guinea,

Page 2/11 
there is very little information available regarding the influence of EVD on health-seeking behaviour post-outbreak. However, in the post-EVD period, a recovery to pre-EVD levels has been reported in reproductive health services and childhood vaccination in the Forest Guinea region $(9,16,17)$. In Guéckédou district, located in Forest Guinea region, all-cause health service visits for children under five years of age were recently reported to have recovered to pre-EVD levels, but the number of malaria cases seen at health centres was still lower than before the EVD outbreak (18). These studies were based on data collected by health facilities and did not investigate the attitudes and experiences of community members themselves. Information on post-EVD health-seeking behaviour and the reasons why some caregivers do not seek health care for their children is essential to guiding post-EVD interventions for community health in terms of prevention, access to care, and psychological well-being. Therefore, this study sought to assess whether EVD memories among community members influence health-seeking behaviour for febrile illnesses in children under five years of age in the post-outbreak period in rural Guinea.

\section{Method}

\section{Study setting}

Guinea is located in West Africa and had a population of 10.5 million in 2014. Most of Guinea's residents are illiterate (67\%), live in a rural setting (71\%) and subsist below the poverty line (55\%) $(12,19)$. The country has thirty-three districts of which twenty-five were affected by the EVD outbreak. The national health system is tiered in primary, secondary, and tertiary levels (20). At the primary level, community healthcare workers (CHWs) provide healthcare and prevention services within communities (21).

The study was conducted in the district of Guéckédou, south-eastern Guinea (Figure 1).. Guéckédou was the epicentre of the EVD outbreak (22)and recorded the highest EVD mortality rate countrywide, with 204 deaths out of 270 confirmed cases (76\%) (2). It was also among the localities that experienced more community resistance to EVD response activities $(4,8,23)$. Furthermore, it belongs to the most malaria-affected natural region in the country where the prevalence of malaria among children under five years of age is $61 \%$ (12). Guéckédou district consists of ten sub-districts and an urban commune.

\section{Operational definition of health-seeking behaviour}

We defined health-seeking behaviour as a "sequence of remedial actions that individuals undertake to rectify perceived illhealth." In this study, we focused on caregivers' decisions regarding the type of healthcare provider patients sought help from, reasons for choice of healthcare professional, and reasons for not seeking help from healthcare professionals (24).

\section{Study design}

This was a cross-sectional study using interview data.

\section{Study participants and sampling}

Caregivers of children under five years of age were interviewed. We considered as caregiver the child's mother or the main person caring for him or her at home. Caregivers were selected through two-stage cluster sampling. In the first stage, the subdistrict with the highest reported EVD case burden (Guèndembou; 60 cases) in Guéckédou and a sub-district with a low reported EVD case burden (Bolodou; one case) were selected. For the second stage, in each selected sub-district, all households with a child aged less than five years who had a fever episode (as reported by the caregiver) within the preceding 30 days were included in the study. The caregiver of one eligible child per household was selected. In households with more than one eligible child, the child who had the most recent episode was selected. All households were visited with the help of local guides, moving from the middle to the ends of each village, clockwise. The visiting process proceeded from the main 
village to the surrounding villages of each sub-district, clockwise, until the desired sample size was reached. In total, fourteen villages (seven in each sub-district) were visited.

The sample size was calculated using Cochran's sample size formula (25), and based on the prevalence of malaria among children under five years in the Forest Guinea region (61\%) (12), a confidence level of $95 \%$ and a margin error of $5 \%$. A minimum of 366 caregivers were needed for the study. Half of the study participants were expected to come from each subdistrict.

\section{Data collection and variables}

Data were collected from 24 September to 4 October 2017 by trained interviewers using Open Data Kit (ODK) with Android mobile phones.

The study variables included: sociodemographic characteristics of caregivers and their children (caregiver's age, education level, marital status, number of household members, main source of household income, household characteristics, age of the child, and gender of the child); EVD-related events and feelings (occurrence of EVD deaths in the household/family, whether EVD impoverished the household/family, fear of shaking hands with friends, fear of hugging friends, fear of sharing plates with friends, fear of hugging household/family members, fear of sharing plates with household/family members, fear of kissing household/family members, preference for washing hands with chlorine solution, keeping chlorine solution at home); interpretation of the child's illness (the child could eat or breastfeed as usual [yes/no], the child could move as usual [yes/no], meaning of the illness to the caregiver, diagnosis of the illness); health-seeking options and reasonssought care [yes/no], if yes: health-seeking place, reasons for selecting the health-seeking place); utilization of health services (if sought care, blood test performed [yes/no], medicines given [yes/no]); and perception of service quality at health facilities as compared with preEVDmedicine availability, antimalarial drug availability, availability of rapid test kits for malaria, waiting time, staff listening to patients, cases left without treatment, staff reliability, facility cleanliness, cost of care, quality of treatment).

\section{Data analysis}

Descriptive variables were presented as proportions or means with standard deviations (SD). Numerical values were assigned to household characteristics by adapting the method developed by the health and demographic survey (12) to measure household property scores. EVD-related feelings were also assigned numerical values to measure EVD outbreak fear among caregivers. Caregivers' level of fear was as assessed using numerical scores, and the maximum fear score had a value of 10. Pearson's chi-square $\left(X^{2}\right)$ and student t-tests were used to compare the variables between the two sub-districts.

A logistic regression using a backward stepwise model was conducted to predict caregivers' high fear score (above the median) and care seeking behaviour. Adjusted odds ratios (AOR) were then derived with $95 \%$ confidence intervals (Cls). The level of significance was set at $p<0.05$.

Data were analysed using SPSS software version 22.0 for Windows (SPSS Inc., Chicago, IL, United States).

\section{Results}

\section{Sociodemographic characteristics of respondents}

The number of households visited in Guèndembou and Bolondou were 219 and 241 respectively. We found eligible children in 196 of the eligible households in Guèndembou and in 202 of the eligible households in Bolodou. Thus, we found that the prevalence of febrile illness in the last 30 days prior to the survey in Guèndembou and Bolondou were $89 \cdot 5 \%$ and $83 \cdot 8 \%$ respectively. 
Overall, 398 caregivers were surveyed. The sociodemographic characteristics differed between the two sub-districts with the exception of the number of household members (Table 1).. Caregivers living in Guèndembou had a higher mean age (33.9 years; SD: 13.9 years) than those in Bolodou (28.7 years; SD: 7.2 years; $p<0.001)$. They were predominantly married or in a union, with a significantly higher proportion of such people in Bolodou (92.1\% ( $n=186)$ compared to $84 \cdot 7 \%(n=166)$ in Guèndembou; $p=0.021)$. In Guèndembou, the majority $(58 \cdot 7 \% ; n=115)$ of caregivers had attended at least primary school, whereas in Bolodou, most caregivers had no education at all $(73 \cdot 8 \% ; \mathrm{n}=149 ; p<0 \cdot 001)$.

\section{EVD- related events and feelings}

More caregivers in Guèndembou $(19.9 \% ; n=39)$ reported the death of family members or friends due to EVD compared to Bolodou (6.9\%; $\mathrm{n}=14 ; P<0.001$ ) (Table 2).. The majority of participants in both districts said that the EVD outbreak had impoverished their families, with a higher proportion in Bolodou $(76 \cdot 2 \%(n=154)$ compared to $64 \cdot 3 \%(n=126)$ in Guèndembou; $p=0.009$ ).

Additionally, more caregivers in Bolodou $(88 \cdot 1 \% ; n=178)$ said they preferred washing their hands with chlorine solution than in Guèndembou (64.3\%; $n=126 ; p<0.001)$; however, the number of those who claimed to keep chlorine solution at home were far more in Guèndembou (55.6\%; $\mathrm{n}=109)$ than in Bolodou $(8.9 \% ; n=18 ; p<0.001)$. In Guèndembou significantly more respondents reported being fearful of shaking hands with friends $(25 \cdot 5 \% ; n=50)$ or hugging friends $(22 \cdot 5 \% ; n=44)$ as compared to $10.9 \%(n=22)$ and $9.4 \%(n=19)$ in Bolodou $(p<0.001)$, respectively. Significantly more caregivers in Guèndembou said that they were fearful of sharing a bed or bed linen $(17 \cdot 3 \% ; n=34 ; p<0 \cdot 001)$ or plates $(16.8 \% ; n=33$; $p<0.001)$ with family members, or of hugging them $(16.8 \% ; n=32 ; p<0.001)$, while the three proportions were all $1 \%(n=2$; $p<0.001)$ in Bolodou. The mean fear score of caregivers was significantly higher in Guèndembou ( $3 \cdot 0 ; S D: 3 \cdot 0)$ than in Bolodou (2.0; SD: 1·1) $(p<0 \cdot 001)$.

\section{Health-seeking options and reasons}

More caregivers sought health care for children with febrile illnesses in the EVD-affected sub-district, i.e., Guèndembou (80.6\%; $\mathrm{n}=158$ ) than in Bolodou (68.8\%; $\mathrm{n}=139 ; p=0.007$ ) (Table 3).. In Guèndembou, 105 of $158(66.5 \%)$ respondents who sought care did so at public health facilities, while in Bolodou 128 of 139 (92.1\%) did so $(p<0 \cdot 001)$.

The primary reason for selecting care facilities was the availability of medicines in both sub-districts $(92 \cdot 4 \%(n=146)$ in Guèndembou and $97 \cdot 1 \%(n=135)$ in Bolodou). The other main reasons in Guèndembou were good staff attitude $(84 \cdot 8 \% ; n=$ 134), availability of staff $(70.9 \% ; n=112)$, and a short waiting time $(79.6 \% ; n=110)$, while the main reasons in Bolodou were low risk of EVD transmission $(64 \cdot 0 \% ; n=89)$ and low risk of EVD misdiagnosis $(63 \cdot 3 \% ; n=88)$.

\section{Perception of health service quality compared with the pre-EVD period}

The majority of caregivers in Guèndembou $(82 \cdot 1 \% ; n=161)$ agreed that more medicines were available at the facilities than during the pre-EVD period, whereas only $29.7 \%(n=60 ; p<0.001)$ agreed with this in Bolodou (Table 4). Opinions regarding health staff availability differed across the two sub-districts; whereas $85.7 \%(n=168)$ of caregivers agreed that more health staff was available at facilities in Guèndembou, only $35.6 \%(n=72 ; p<0.001))$ agreed with this in Bolodou. In Guèndembou, 70 of $196(35 \cdot 7 \%)$ respondents agreed that the cost of health care was less expensive than during the pre-EVD period, whereas only 14 of $202(6.9 \%)$ in Bolodou agreed with this $(p<0.001)$.

\section{Factors associated with seeking care}

A bivariate analysis was conducted to examine factors that were associated with seeking health care for children under-five years of age (Table 5).. Factors that were significantly associated with seeking care included residing in Guèndembou, death 
of a family member or friend due to due to EVD, perceived greater quantity of medicines at health facilities in the post-EVD period, perceived better treatment at health facilities post-EVD, and perceived less cost of care at health facilities post-EVD. Although it was not statistically significant, caregivers with a fear score above the median were 1.68 times more likely to seek care than those whose fear score was equal to or below the median.

In the multiple-logistic regression analysis (Table 6), only residing in Guèndembou (AOR = 1.74; $95 \% \mathrm{Cl}: 1 \cdot 09-2 \cdot 79)$ was positively associated with seeking care. Though not statistically significant, caregivers who reported family members' or friends' death due to EVD were also more likely to seek care $(\mathrm{AOR}=2 \cdot 12 ; 95 \% \mathrm{Cl}: 0 \cdot 91-4 \cdot 91)$.

\section{Discussion}

This study revealed that a number of community members in the rural district of Guéckédou still live with fear related to EVD nearly two years after the outbreak, and that the majority of community members sought care for their children with febrile illnesses in the post-EVD period. The findings also suggested that EVD memories might still affect community members' health-seeking behaviour. Even though not statistically significant, caregivers who had higher EVD-related fear scores and those who reported the death of family members or friends due to EVD were more likely to seek care.

Indeed, we were expecting to find a negative impact of the EVD outbreak on post-outbreak health-seeking behaviour for febrile illnesses. Previous studies on post-EVD health service utilization in Guinea reported recovery gaps demonstrating that healthseeking behaviour was lower in 2016 compared to the pre-EVD period $(9,16-18)$. The present findings reflect a gradual improvement in health service utilization after the EVD outbreak. Dunbar et al. reported that the malaria program in Liberia could require 26 months after the acute phase of the EVD outbreak to recover to pre-EVD levels (26). Improvement in utilization of health services could be explained by two factors. First, there is a perceived improvement of health service quality by communities, as shown by this study, in the post-EVD compared to the pre-EVD periods. As part of an effort to strengthen the health system during the post-EVD period, the Guinean government and international organizations might have undertaken initiatives to improve the quality of and access to health services (21). Second, a gradual regaining of community confidence in the health system could have been achieved after the EVD outbreak. In a post-outbreak momentum to improve a health system that had been shaken by a recent history of community mistrust due to the outbreak, it is likely that priority would be given to the rebuilding of the community's trust in the health system.

The study findings also showed positive and negative influences of the EVD outbreak on community members' post-EVD attitudes. As positive influence, people were more likely to utilize chlorine for hand hygiene. In fact, more than half of the caregivers in Guèndembou keep chlorine at home, implying that these individuals are aware of its importance. Furthermore, the majority of caregivers in both sub-districts prefer to wash their hands with chlorine. These attitudes show the positive perception of rural communities toward chlorine and afford an opportunity to further encourage its use. This would improve and sustain infection prevention measures in these communities and prevent hygiene-related diseases among Guinean children, especially in rural settings $(12,20)$. However, Somparé questioned the community's will to continue systematic hand washing with chlorine in the long run given that this practice might trigger negative memories of the EVD outbreak. Some community members might even reject any symbolic behaviour or practices related to EVD (27). This suggests the need for appropriate strategies involving social and health scientists to sustain infection prevention practices implemented in communities during the EVD crisis.

As a negative influence, we found that residents of Guinean rural communities live with a persistent fear of EVD contamination that affects their social relationships. For instance, despite this being one of the community's key values, a number of caregivers refrain from shaking hands with or hugging friends or family members. In a context where cultural values prevail as is the case in Guinea (28), such attitudes reflect a sociocultural impact of the EVD outbreak and are more likely to weaken social ties in rural Guinea. In addition, this could reflect a psychosocial effect of EVD on individuals since people's negative memories of EVD-related deaths and stigma could drive such attitudes. This suggests the need for refined health education strategies at the community level in a manner that contributes to preserving the community's key values. It

Page 6/11 
also calls for further research on the psychosocial effects of EVD in Guinean communities in order to restore individuals' sense of psychosocial well-being. Shanahan reported that many bereaved people and orphans from the EVD outbreak were exposed to complex grief and stress after the outbreak in Sierra Leone and urged health workers to address their psychosocial needs (29).

More caregivers sought health services for their children in the EVD-affected sub-district of Guèndembou than in the less affected sub-district of Bolodou. This result may suggest the possibility of a greater commitment of health system actors to improving health service utilization in heavily EVD-affected areas. For instance, health facilities might have been better equipped with quality services to deal with the outbreak in the heavily EVD-affected areas than in the less affected areas. In the present study, improvement of health services following the outbreak was perceived by more caregivers in Guèndembou (most affected by EVD) than Bolodou (less affected). Additionally, with the presence of a private clinic in Guèndembou that offers the community the opportunity to receive care on credit, it is possible that more caregivers are seeking health services in that sub-district than in Bolodou. This emphasizes the disparities in access to and quality of post-EVD health services across sub-districts in the country, especially for child febrile illnesses. Given that Guinea is malaria-endemic, with a high prevalence of malaria among children under the age of five ( $44 \%$ nationwide and $61 \%$ in the region including the study site) (12), many children suffering from malaria might still not access appropriate malaria treatment in number of rural areas. However, the National Malaria Control Program has recruited more community health workers and improved the supply of malaria drugs and diagnostic tests to improve children's access to malaria treatment in the post-EVD period (21).Despite these improvements, health disparities across local settings have been reported to often be hidden by the improvements shown by national/global indicators (30). Therefore, both EVD-affected and less affected sub-districts should be a priority for health service quality surveys and the improvement of malaria activities in the post-outbreak period.

A few limitations to this study should be noted. First, the comparison groups-the sub-districts as well as caregivers across the sub-districts-were not directly comparable; this created the risk of potential bias in estimating the effect of the EVD outbreak on health-seeking behaviours. Second, the study was limited to two sub-districts within a single district and therefore could not address the situation in the other EVD-affected districts in the country. However, our data allowed for a comparison between an EVD-affected sub-district and the least affected sub-district. The study should therefore be relevant for districts with similar EVD epidemiological contexts.

\section{Conclusion}

This study found that community members in the rural district of Guéckédou still live with fear related to EVD nearly two years after the outbreak. It also demonstrated that EVD memories are likely to have a positive influence on health seeking behaviour. Findings revealed the opportunities that the EVD outbreak provide regarding an improvement in infection prevention measures, but also call for more efforts in the health domain to preserve communities' key values and address the psychosocial effect of EVD in rural Guinea. Finally, the study advocates addressing disparities in the access of children to quality care across sub-districts especially for febrile illnesses such as malaria.

\section{Declarations}

\section{Ethics approval and declaration to participate}

The study protocol was approved by the National Ethical Committee for Health Research of Guinea (Ref 068/CNERS/17). It also fulfilled the requirements of the Ethical Review Board of the Institute of Tropical Medicine, Nagasaki University, Japan (Ref 170707167).

Verbal informed consent was obtained for each participant prior to data collection and data were anonymized prior to analysis. 


\section{Consent for publication}

Not applicable

\section{Availability of data and materials}

The datasets used and/or analysed during the current study are available from the corresponding author on reasonable request.

\section{Competing interests}

The authors declare that they have no competing interests.

\section{Funding}

This study was partially funded by the School of Tropical Medicine and Global Health, Nagasaki University, Japan. The deficit part was paid by the authors.

\section{Authors' contributions}

BSC and AD did the literature search. BSC and JO were involved with conception and design of the protocol which was reviewed by $A D$ and all three authors were involved with acquisition of data. BSC and JO did the data analysis and all authors were involved with interpretation. The first draft of the manuscript was written by BSC and critically reviewed by JO and AD. All authors have given approval for the final version to be published and are accountable for its content.

\section{Acknowledgements}

We thank Delphin Kolie, Moussa Douno, Bernard Kondiano, Christine Songbono, and Norbert Tolno for their help with the data collection. We are grateful to the District Health Office of Guéckédou, community leaders, and the local guides for facilitating the data collection. Thanks to the respondents who offered their time for the interviews.

\section{List of abbreviation}

AOR:

Ajusted odd ratio

CHWs :

Community health workers

$\mathrm{Cl}$ :

Confidence interval

EVD:

Ebola virus disease

$S D$ : 


\section{References}

1. World Health Organization Ebola Response Team. After Ebola in West Africa-Unpredictable Risks, Preventable Epidemics. N Engl J Med. 2016;375(6):587-96.

2. Ministère de la Santé et Organisation Mondiale de la Santé. Rapport de la situation epidémiologique maladie à virus Ebola en Guinée. Rapport du 01 Janvier 2016. [Epidemiological situation report of Ebola virus disease in Guinea. 01 January 2016 Report.]. 2016.

3. Fribault M. Ebola en Guinée: Violences historiques et régimes de doute. [Ebola in Guinea: historical violences and regimes of doubt.]. Anthropologie \& Santé. 2015.

4. Fairhead J. Understanding social resistance to Ebola response in Guinea. Afr Stud Rev. 2016;59(3):7-31.

5. Thiam S, Delamou A, Camara S, Carter J, Lama EK, Ndiaye B, et al. Challenges in controlling the Ebola outbreak in two prefectures in Guinea: why did communities continue to resist? Pan Afr Med J. 2015;22(Supp 1):22.

6. Shrivastava S, Shrivastava P, Ramasamy J. Ebola disease: Infection prevention and control in hospital and community settings. Iran J Nurs Midwifery Res [Internet]. 2015;20(4):526. Available from: http://www.ijnmrjournal.net/text.asp? 2015/20/4/526/161010

7. Wilkinson A, Fairhead J. Comparison of social resistance to Ebola response in Sierra Leone and Guinea suggests explanations lie in political configurations not culture. Crit Public Health [Internet]. 2017;27(1):14-27. Available from: http://dx.doi.org/10.1080/09581596.2016.1252034

8. Anoko J. Communication with rebellious communities during an outbreak of Ebola virus disease in Guinea: An anthropological approach. Ebola Response Anthropol Platf [Internet]. 2014;1-21. Available from: http://www.ebolaanthropology.net/wp-content/uploads/2014/12/Communicationduring-an-outbreak-of-Ebola-Virus-Disease-withrebellious-communities-in-Guinea.pdf

9. Camara BS, Delamou A, Diro E, Béavogui AH, El Ayadi AM, Sidibé S, et al. Effect of the 2014/2015 Ebola outbreak on reproductive health services in a rural district of Guinea: An ecological study. Trans R Soc Trop Med Hyg. 2017;111(1).

10. Delamou A, Delvaux T, El Ayadi AM, Beavogui AH, Okumura J, Van Damme W, et al. Public health impact of the 20142015 Ebola outbreak in West Africa: Seizing opportunities for the future. BMJ Glob Heal. 2017;2(2):e000202.

11. Plucinski MM, Guilavogui T, Sidikiba S, Diakité N, Diakité S, Dioubaté M, et al. Effect of the Ebola-virus-disease epidemic on malaria case management in Guinea, 2014: A cross-sectional survey of health facilities. Lancet Infect Dis. 2015;15(9):1017-23.

12. Institute of Statistics. Guinea Demographic and Health and Multiple Indicators Survey 2012 [Internet]. 2013. Available from: http://www.stat-guinee.org/PUB/rapports/INS_RGPH_2014_decret.pdf

13. Friedman A, Cobb G, Hudson A, Morrissette D, Saindon J, Bird B, et al. CDC responder stories-Health communication in Guinea and Atlanta [Internet]. CDC. 2015. Available from: https://www.cdc.gov/vhf/ebola/hcp/stories-healthcommunications-in-guinea-atlanta.html

14. United Nations Development Group. Socio-Economic Impact of Ebola Virus Disease in West African Countries. 2015.

15. Agüero JM, Beleche T. Health shocks and their long-lasting impact on health behaviors: Evidence from the 2009 H1N1 pandemic in Mexico. J Health Econ [Internet]. 2017;54:40-55. Available from:

http://dx.doi.org/10.1016/j.jhealeco.2017.03.008

16. Camara BS, Delamou A, Diro E, El Ayadi AM, Beavogui AH, Sidibé S, et al. Influence of the 2014-2015 Ebola outbreak on the vaccination of children in a rural district of Guinea. Public Heal Action. 2017;7(2):161-7.

17. Delamou A, Ayadi AM El, Sidibe S, Delvaux T, Camara BS, Sandouno SD, et al. Effect of Ebola virus disease on maternal and child health services in Guinea: a retrospective observational cohort study. Lancet. 2017;5(17):1-10. 
18. Kolie D, Camara BS, Delamou A, Beavogui AH, Hermans V, Edwars JK, et al. The Ebola-effect in Guinea 2014-15: Tangled trends of malaria care in children under-five. PLoS One. 2018;13(2):e0192798.

19. Présidence de la Republique. Troisième recensement général de la population et de l'habitat (RGPH), 2014, République de Guinée. [Third General Census of Population and Housing (RGPH), 2014, Republic of Guinea.] [Internet]. 2015. Available from: http://www.stat-guinee.org/PUB/rapports/INS_RGPH_2014_decret.pdf

20. Ministère de la Santé de Guinée. Annuaire des statistiques sanitaires nationales 2012. [National health statistics yearbook 2012]. Conakry; 2015.

21. Ministère de la Santé de Guinée. Plan stratégique national de lutte contre le paludisme 2013-2017. Programme National de Lutte contre le Paludisme. [National strategic plan for malaria control 2013-2017. National Malaria Control Program]. 2014.

22. World Health Organization. Origins of the 2014 Ebola epidemic [Internet]. 2001 [cited 2019 May 17]. Available from: www.who.int/csr/disease/ebola/one-year-report/virus-origin/en/

23. WHO Guinea. Ebola en Guinée: la Communication, une pièce maîtresse pour endiguer l'épidémie [Internet]. 2015 [cited 2017 Dec 27]. Available from: http://www.afro.who.int/fr/news/ebola-en-guinee-la-communication-une-piece-maitressepour-endiguer-lepidemie

24. Mahmood SS, Iqbal M, Hanifi SM. Health-seeking Behavior [Internet]. Health for the Rural Masses. 2009. 1-130 p. Available from:

https://assets.publishing.service.gov.uk/media/57a08b63ed915d622c000c65/FHSbookHealthforRuralMasses.pdf\#page $=74$

25. Statistics How To. The Cochran's sample size formula [Internet]. 2017 [cited 2017 Jul 22]. Available from: http://www.statisticshowto.com/probability-and-statistics/find-sample-size/

26. Dunbar N., Richards E., Woldeyohannes D, Van den Bergh R, Wilkinson E, Tamang D, et al. Knockdown and recovery of malaria diagnosis and treatment in Liberia during and after the 2014 Ebola outbreak. Pha. 2017;7(S1):S76-81.

27. Somparé AW. La politique et les pratiques de santé en Guinée à l' épreuve de l' épidémie d' Ebola: Le cas de la ville de Conakry. [ Health policy and practice in Guinea facing the Ebola epidemic: The case of the city of Conakry.]. Santé Polit urbaines. 2017;78.

28. Barry AAB. Interpreting the health, social, and political dimensions of the Ebola crisis in Guinea. In: Abdullah I, Rashid I, editors. Understanding West Africa's Ebola epidemic Towards a political economy. Zed Books. London; 2017. p. 70-84.

29. Shanahan F. Prioritizing psychosocial support for people affected by Ebola in Sierra Leone. Recomm Ebola Recover Pledging Conf New York City,. 2015;1-6.

30. Thomson DR, Amoroso C, Atwood S, Bonds MH, Rwabukwisi FC, Drobac P, et al. Impact of a health system strengthening intervention on maternal and child health outputs and outcomes in rural Rwanda 2005-2010. BMJ Glob Heal [Internet]. 2018;3(2):e000674. Available from: http://gh.bmj.com/lookup/doi/10.1136/bmjgh-2017-000674

\section{Tables}

Due to technical limitations, Tables 1-6 are only available for download from the Supplementary Files section.

\section{Figures}



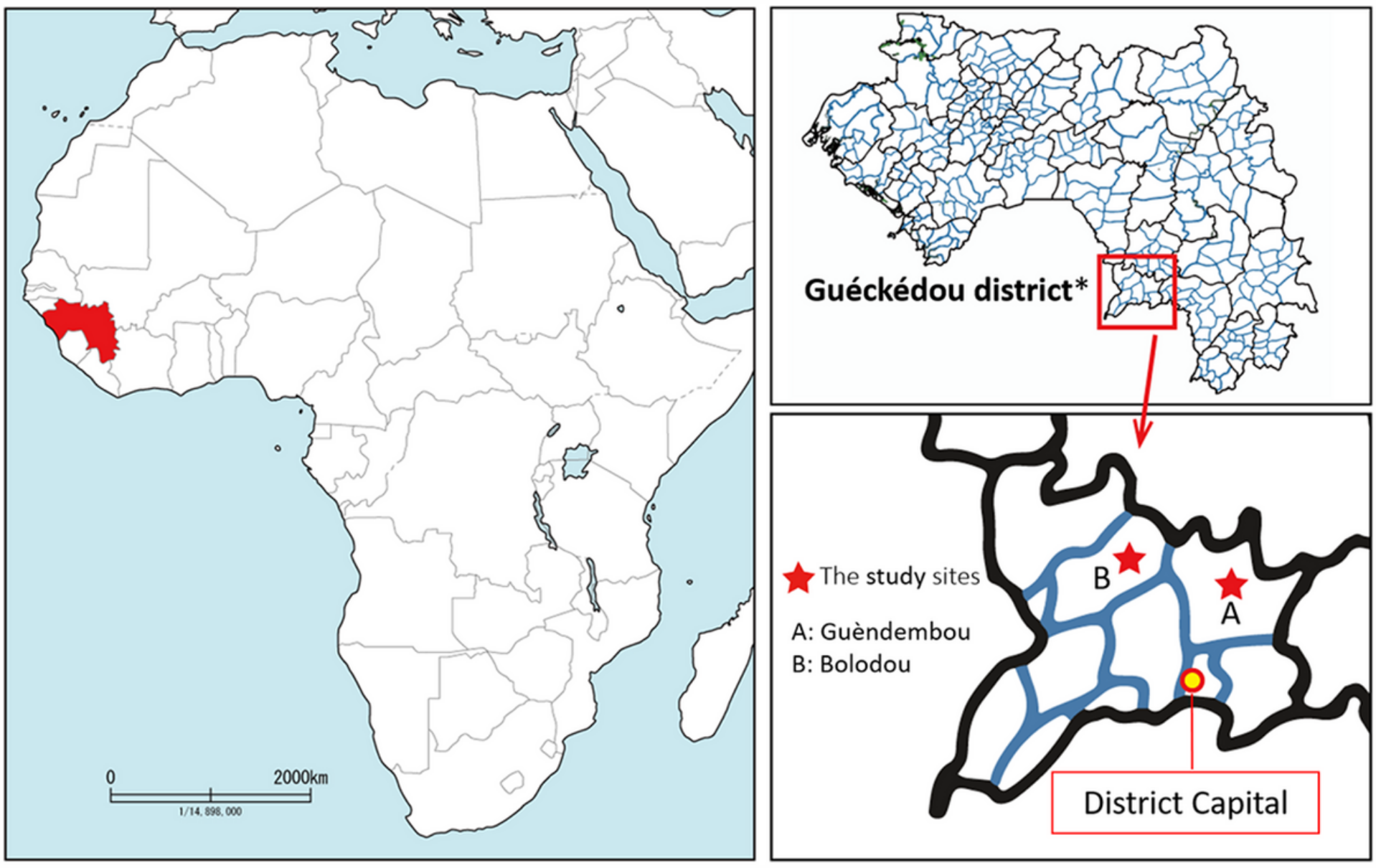

Figure 1

Map of the study setting - * Guéckédou district is an EVD epicentre and prevalence of malaria among children under five years of age in this district was $61 \% .12$ The number of reported EVD cases in Guèndembou (A) and Bolodou (B) was 60 and 1 respectively.

\section{Supplementary Files}

This is a list of supplementary files associated with this preprint. Click to download.

- supplement1.pdf

- supplement2.pdf

- supplement2.pdf

- supplement4.pdf

- supplement5.pdf

- supplement6.pdf 